\title{
'Mini-GEMs': short, focused e-learning videos in geriatric medicine
}

\author{
Authors: Mark Garside, ${ }^{A}$ James Fisher, ${ }^{B}$ Kelly Hunt ${ }^{A}$ and Peter Brock ${ }^{A}$
}

\section{Aims}

To develop a library of free open-access e-learning resources in geriatric medicine, based on the Pecha Kucha presentation format.

\section{Methods}

Pecha Kucha (Japanese for 'chit-chat') is a presentation style and format that was developed in the early 2000s, but has gained widespread popularity over the last few years. It specifies that speakers should use 20 slides for 20 seconds each, with each presentation therefore lasting a total of 6 minutes and 40 seconds. This ensures that speakers are focused and concise. We believed that this format would translate well into the context of medical education, as an alternative to more lengthy online learning resources that already exist. As a result, we have created a small but growing library of video podcasts that we call 'Mini-GEMs' (geriatric e-learning modules). These are aimed at junior doctors and allied health professionals who care for older patients.

Each mini-GEM covers a very specific clinical topic, and lasts no longer than 7 minutes (the duration of an average OSCE station). With engaging speakers and integration of multimedia and by keeping text and bullet points to a minimum, the videos are easy to watch, listen to and understand. Learning outcomes are clearly stated at the beginning and end of each mini-GEM.

Hosting the mini-GEMs on YouTube ensures compatibility with the majority of computers and internet-connected mobile devices, allowing the videos to be viewed on a smartphone or tablet. No login or registration is required, and the emphasis is on accessibility and ease of use. The relatively short length of the videos means that learning can happen 'on the go'.

\section{Results}

After 9 months, we have created a library of 10 videos, with over 2,000 views from 66 different countries ( $74 \%$ of views within the UK), and over 6,800 minutes of content watched. Informal feedback from social media users has been overwhelmingly positive, and more formal evaluation of this format of e-learning is planned.

\section{Conclusions}

In a digitally connected world containing an overwhelming amount of detailed and extensive information, there is an appetite amongst busy clinical staff for short, focused, clinically relevant, video-based online medical updates.

\section{Conflict of interest statement}

None. 